\title{
Educación virtual y su impacto socio - económico en los estudiantes y docentes de una unidad educativa
}

\section{Virtual education and its impact partner - economic in the educational students and of an educative unit}

Hólguer Rodrigo Altamirano P. ${ }^{1}$, Vilma Janeth Cadena P. ${ }^{2}$ \& Bella Eloísa Arias V. ${ }^{3}$

\begin{abstract}
The pandemic caused by Covid -19 has left consequences of high impact in all the fields of the society and the education is not free of this impact. Therefore, the educative managers and leaders have had to redefine their practices and models doing it virtual and using the technology by means of the different platforms from the communication. Objective. To define with clarity the impacts at economic and social level in the educational families and of an educative unit, to use different suitable resources and to promote the adaptation to the new educative system and to obtain better advantage of the education in these more vulnerable sectors of the society. Methodology. The paradigms by the type of study were applied, that it requires like instrument of data collection, the survey by means of google forms by the present conditions that do not allow a field study. 2 surveys were made, first second a 31 of plant of the educative unit in study took place to 198 parents of educational family and. As tool to process the data were used SPSS version 24. Results. A strong affectation in the students is observed when having a learning level that goes between the $51 \%-75 \%$, thus agreeing educational parents and when assuring that they would like to return to the actual modality; educational $61 \%$ of and $55 \%$ of students have problems of connection by the Internet; it has had to invest in the purchase of some computer for the virtual classes; stress is the

\footnotetext{
${ }^{1}$ Instituto Tecnológico Superior Bernardo O'Higgins, Docente Ciencias Económicas, Quito, Ecuador. holguer_rodrigo@yahoo.com ORCID: https://orcid.org/0000-0002-9368-6173

2 Unidad Educativa Oswaldo Guayasamín, Rectora y Docente, Santo Domingo, Ecuador. vilma_janeth.cadena@ hotmail.com ORCID: https://orcid.org/0000-0003-3599-575X

3 Contadora Profesional CPA. Santo Domingo, Ecuador. bella.arias1710@gmail.com ORCID: https://orcid.org/0000-0002-3317-5486
} 
main problem for students with educational $27 \%$ and with $31 \%$ and basically in the case of the students in its free times they are dedicated to tasks of the house or of using the cellular one not with educative aims but through games that generate addiction in youth. Conclusion. The pandemic has generated a new form of education as much for the families as for the educational ones and shows the adaptation capacity before a scene that had to arrive in gradual and nonsudden form.

Keywords: virtual education; impact economic partner; educative units; pandemic, behaviors.

\section{Resumen}

La pandemia provocada por la Covid -19 ha dejado consecuencias de alto impacto en todos los campos de la sociedad y la educación no está exenta de dicho impacto. Por lo tanto, los gestores y líderes educativos han tenido que redefinir sus prácticas y modelos haciéndola virtual y utilizando la tecnología mediante las diferentes plataformas de la comunicación. Objetivo. Definir con claridad los impactos a nivel económico y social en las familias y docentes de una unidad educativa, para utilizar diferentes recursos idóneos y promover la adaptación al nuevo sistema educativo y lograr mejor aprovechamiento de la educación en estos sectores más vulnerables de la sociedad. Metodología. Se aplicaron los paradigmas positivista e interpretativo por el tipo de estudio, que requiere como instrumento de recolección de datos, la encuesta mediante google forms por las condiciones actuales que no permiten un estudio de campo. Se realizaron 2 encuestas, la primera se efectuó a 198 padres de familia y la segunda a 31 docentes de planta de la unidad educativa en estudio. Como herramienta para procesar los datos se utilizó SPSS versión 24. Resultados. Se observa una fuerte afectación en los alumnos al tener un nivel de aprendizaje que va entre el 51\%-75\%, coincidiendo así padres y docentes al asegurar que les gustaría retornar a la modalidad presencial; el $61 \%$ de docentes y el 55\% de estudiantes tienen problemas de conexión por el internet; se ha tenido que invertir en la compra de algún computador para las clases virtuales; el estrés es el problema principal para estudiantes con el $27 \%$ y docentes con el $31 \%$ y básicamente en el caso de los estudiantes en sus tiempos libres se dedican a tareas de la casa o utilizar el celular no con fines educativos sino a través de juegos que generan adicción en la juventud. Conclusión. La pandemia ha generado una nueva forma de educación tanto para las familias como para los docentes y pone de manifiesto la capacidad de adaptación ante un escenario que debía llegar en forma paulatina y no repentina.

Palabras claves: educación virtual; impacto socio económico; unidades educativas; pandemia, comportamientos. 


\section{Introducción}

Antecedentes:

“A finales del 2019 se suscitó un brote de un cuadro clínico caracterizado por fiebre, tos seca y debilidad generalizada, acompañado en ocasiones de síntomas gastrointestinales, en un mercado mayorista de mariscos de Huanan, en Wuhan, China. El agente causal de este brote fue identificado como un novel beta coronavirus, al que se le denominó 2019 nuevo coronavirurs (2019n-CoV). El 11 de febrero del 2020 el comité internacional sobre taxonomía de virus le designó como síndrome respiratorio agudo severo coronavirus 2 (SARS-Cov-2); mientras que el cuadro clínico fue oficialmente llamado coronavirus disease-2019 (COVID-19) (...) Además que ha habido transmisión interpersonal fuera de Wuhan e incluso de China, por lo que acordó en que el brote cumple con los criterios para declarar una emergencia de salud pública de importancia internacional” (Inca Ruiz \& Inca León, 2020).

Consecuente con los cambios en la educación, de presencial a virtual son los avances de las tecnologías de la información y comunicación tanto para estudiantes como docentes y que han permitido que gran parte de la población se capacite y pueda enfrentar el desafío de la profesionalización virtual. (Suárez Huz, 2018). El proceso de formación de las generaciones actuales en un mundo globalizado requiere la adaptación a nuevas herramientas como: tabletas, computadoras, celulares, etc, lo que se puede evidenciar en el sistema educativo con el uso de plataformas virtuales unas mejor que otras en temas de amigabilidad, una especie de alfabetización digital para las generaciones de hoy. (Mujica, 2020)

Las consecuencias de esta pandemia, comenzó en Ecuador al identificarse el primer caso el 29 de febrero del 2020 y que sería el inicio de los efectos de la mortal pandemia hasta la fecha. Uno de los efectos fue el cambio en la educación presencial al experimentar una transformación producto de aspectos externos que resultaron incontrolables, en complemento con las decisiones de las autoridades al imponer el confinamiento social. "El aprendizaje es un hecho tan cotidiano que nos lleva a pensar que azarosa y sistemáticamente se aprende una nueva habilidad o un nuevo concepto; sin embargo, si se observa con cuidado las situaciones en que se aprende, se apreciará que el aprendizaje no es tan fortuito como parece". (Jama Zambrano \& Cornejo Zambrano, 2016, p.110)

Para conseguir que la educación llegue a todos, el gobierno "garantizará el acceso universal, permanencia, movilidad y egreso sin discriminación alguna y la obligatoriedad en el nivel inicial, básico y bachillerato o su equivalente" (Asamblea Nacional del Ecuador, 2018, p. 18) razón por la que destina del presupuesto general del estado recursos económicos como se puede apreciar en el Gráfico 1: 
Gráfico 1. Evolución del gasto de educación con respecto al PIB

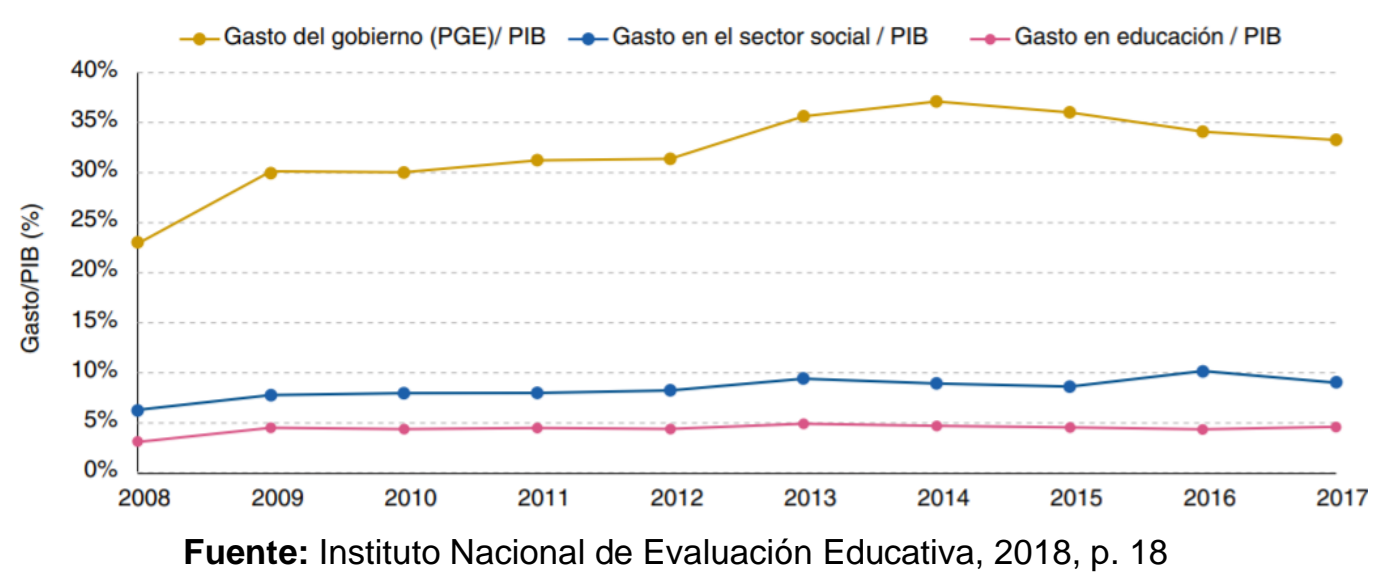

Históricamente se ha planteado en el presupuesto general del estado que el porcentaje destinado a educación sea al menos del $0.5 \%$ del PIB hasta alcanzar el $6 \%$ del PIB. Para el año $2019^{4}$ se redujo la asignación al $4.55 \%$ del PIB equivalentes a $\$ 4.887,42$ millones de dólares mientras que para el año 2020 la asignación en el presupuesto aprobado fue de $\$ 5.565,22$ y terminó con una reducción de $\$ 996^{5}$ millones de dólares. Correspondería analizar los informes de las autoridades sobre el verdadero uso de los mismos y cuál va a ser la asignación para el año 2021 en educación en un período de cambio presidencial con problemas económicos para los próximos 4 años.

Al analizar el triángulo de la virtualidad: docentes, estudiantes y familias, es imprescindible entender cada elemento por separado y luego juntarlos para determinar su importancia en la sociedad educativa. En el caso del docente, el "diseñar la práctica pedagógica no sólo exige ordenar sus componentes para ser aprendidos por los alumnos, sino prever las condiciones de la enseñanza en el contexto educacional o fuera de él. La función más inmediata que deben desarrollar los docentes ${ }^{6}$ es la de diseñar o prever la práctica de la enseñanza” (Castillo, 2010, pág. 903). Ahora según el portal Ecured (s.f.) un estudiante ${ }^{7}$ es la "persona que cursa estudios en un centro docente, especialmente de enseñanza media o superior. Estudiante es la palabra que permite referirse a quienes se dedican a la aprehensión, puesta en práctica y lectura de conocimientos sobre alguna ciencia, disciplina o arte". La tercera arista es la familia, que es considerada como "una comunidad de amor y de solidaridad insustituible para la enseñanza y transmisión de los valores culturales, éticos, sociales, espirituales y religiosos, esenciales para el desarrollo y bienestar de los propios miembros y de la sociedad" (Scola, 2012)

\footnotetext{
${ }^{4}$ De acuerdo al Informe del Presupuesto General del Estado: (Ministerio de Economía y Finanzas, 2020, pág. 36)

${ }^{5}$ El lector puede investigar más en: Primicias (2020)

${ }^{6}$ Para el Ministerio de Educación de Ecuador, se define al docente como:" "Personas oficialmente habilitadas - en régimen de empleo pleno o parcial - para orientar y encauzar la experiencia de aprendizaje de estudiantes, cualquiera que sea su certificación profesional o la modalidad de enseñanza (por ejemplo, presencial o a distancia)" (Ministerio de Educación del Ecuador, 2013, p. 8).

7 Para el Ministerio de Educación de Ecuador, se define al estudiante como: "Es toda persona que demanda servicios educativos y concurre a las aulas, dentro de lo programado por las IE" (Ministerio de Educación del Ecuador, 2013, pág. 8).
} 
El elemento común entre ellos es la educación y "lo cierto es que entre familias y establecimientos educativos debería desarrollarse una relación colaborativa, una relación de sociedad o alianza (partnership) entre educadores, padres y otros actores de la comunidad, en la que compartan la responsabilidad por el aprendizaje" (Razeto, 2016). Esto considerando que el cambio de modalidad de educación puede provocar condiciones que afectan la estabilidad emocional del estudiante y del docente si no se consideran medidas al respecto, por lo que resulta un reto importante para el docente estar preparado para el desafío tecnológico (Gómez \& Álvarez, 2020)

\section{Objetivo}

Definir con claridad los impactos a nivel económico y social en las familias y docentes de una unidad educativa, para utilizar diferentes recursos idóneos y promover la adaptación al nuevo sistema educativo y lograr mejor aprovechamiento de la educación en estos sectores más vulnerables de la sociedad.

\section{Justificación}

Es importante considerar que las familias han experimentado cambios en su ambiente natural tanto en el trabajo como en la educación y sobre todo en aspectos económicos y sociales producto de la educación virtual.

A esto se añade que estudiantes y docentes deban usar plataformas que requieren de computadores o tabletas que se complementarían con una buena velocidad de internet para llevar a cabo una sesión virtual sin inconvenientes.

Este desafío no solo es para las familias sino también para los docentes, cuya capacidad de adaptación en sus hogares han sido puestas al límite, en unos casos por la velocidad del internet y en otros por el conocimiento escaso en el manejo de las tecnologías de la información y comunicación TIC`s sobre todo para aquellos docentes tradicionalistas que son resistentes al uso de herramientas virtuales en pleno siglo XXI.

\section{Metodología}

El paradigma utilizado es de tipo positivista para el enfoque cuantitativo y para el enfoque cualitativo el paradigma utilizado fue el Interpretativo, por la naturaleza de la información que se obtuvo.

La información se basó en el método inductivo - deductivo por las características importantes de la población objetivo. La variable dependiente de estudio está relacionada a la educación virtual y las variables explicativas son los impactos económicos y sociales en los hogares de los estudiantes y docentes de la unidad educativa en estudio de investigación.

Se utilizó la encuesta como instrumento para recopilar datos a los padres de familia y a los docentes y su validez se determinó en la realización de pruebas piloto que 
confortaron como válida a esta herramienta y en la revisión de dos expertos en el área social y económica.

Se realizaron dos encuestas, la primera a los padres de familia de la unidad educativa y la segunda a la totalidad del cuerpo docente.

En el primer caso constan 15 preguntas, divididas en 3 dimensiones: educación virtual, impacto económico e impacto social. Para el caso de la segunda encuesta a los docentes, consta de 14 preguntas bajo las mismas dimensiones.

La relación que existe entre las dos encuestas se puede determinar en la tabla 1

Tabla 1. Dimensiones de la encuesta a padres de familia y docentes de la unidad educativa

FAMILIAS DE LOS ESTUDIANTES

\begin{tabular}{l}
\hline Dimensión \\
\hline Educación Virtual \\
Aprendizaje Virtual \\
Tipo de Educación \\
Dificultad en Clase \\
Impacto Económico \\
Inversión en equipos \\
Apoyo gubernamental \\
Ingresos Familiares \\
Impacto Social \\
Compartir espacios \\
Apoyo en clases \\
Cambios del alumno \\
Actividades post - clases
\end{tabular}

DOCENTES

\begin{tabular}{|c|c|c|}
\hline Items & Dimensión & Items \\
\hline $1,2,3,4,5$ & $\begin{array}{l}\text { Educación Virtual } \\
\text { Aprendizaje Virtual } \\
\text { Tipo de Educación } \\
\text { Dificultad en Clase }\end{array}$ & $\begin{array}{l}1,2,3,4, \\
5,6\end{array}$ \\
\hline $\begin{array}{l}6,7,8,9, \\
10,11\end{array}$ & $\begin{array}{l}\text { Impacto Económico } \\
\text { Inversión en equipos } \\
\text { Otros Ingresos } \\
\text { Ingresos Familiares }\end{array}$ & $7,8,9,10$ \\
\hline $\begin{array}{l}12,13,14, \\
15\end{array}$ & $\begin{array}{l}\text { Impacto Social } \\
\text { Compartir espacios } \\
\text { Apoyo en clases } \\
\text { Cambios del docente } \\
\text { Actividades post - cla }\end{array}$ & $\begin{array}{l}11,{ }^{12}, \\
13,14\end{array}$ \\
\hline
\end{tabular}

Fuente: Elaboración de los autores

Para una mejor comprensión del lector, a las preguntas de las dos encuestas, se las ha categorizado así:

PP: Pregunta al padre de familia

PD: Pregunta al docente

\section{La Población y muestra}

La población total de estudiantes fue de 375 estudiantes, los cuales fueron sometidos al cálculo del tamaño de muestra:

$$
\eta=\frac{Z^{2} p^{*} q * N}{e^{2}(N-1)+Z^{2} * p^{*} q}
$$

Al aplicar la fórmula se determinó una muestra de 190 estudiantes; sin embargo, se pudo recopilar en el estudio 198 casos por correspondencia que cada hogar se ve 
representado por un estudiante, la muestra se calculó con un error del 5\% y un nivel de confianza del $95 \%$.

En el análisis final realizado, se pudo determinar un total de 198 encuestas efectivas por medio de la plataforma Google Forms.

Los estudiantes comprenden una población de: 100 mujeres y 98 hombres de la Unidad Educativa "Oswaldo Guayasamín". En la segunda encuesta, se pudo analizar la población de 31 docentes de la Unidad Educativa y correspondió a 10 mujeres y 21 hombres, como se puede ver en el gráfico 2

Gráfico 2. Población de estudiantes y docentes de la unidad educativa

\begin{tabular}{|l|l|l|}
\hline $\begin{array}{c}\text { Composición de la Población Estudiantil } \\
\text { clasificada según el sexo }\end{array}$ & $\begin{array}{c}\text { Composición de la Población Docente } \\
\text { clasificada según el sexo }\end{array}$ \\
\hline $49 \%$ & "Femenino - Masculino \\
\hline
\end{tabular}

Fuente: Encuesta realizada por los autores

Se puede evidenciar que en la estructura poblacional de estudiantes se encuentra equilibrada en cuanto a equidad de género, mientras que la población docente muestra una clara concentración sobre el sexo masculino con una brecha de casi el doble respecto a la población femenina.

\section{Resultados}

La población estudiantil se clasificó por grados, el gráfico 3 muestra esta composición, en lo que se destaca que la mayor concentración de estudiantes se produce en primero de bachillerato con 61 estudiantes, posteriormente el segundo de bachillerato con 47 estudiantes como los dos grados con mayor representación estudiantil.

Gráfico 3.Población de estudiantes de la unidad educativa

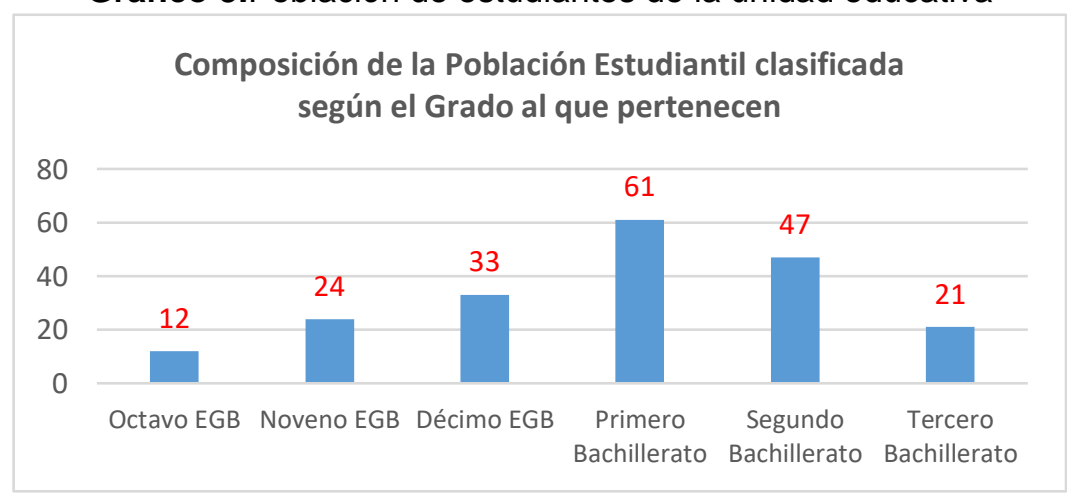

Fuente: Encuesta realizada por los autores 
La garantía de la educación está en la calificación académica de los docentes, el gráfico 4 se pueden apreciar que 22 docentes tienen calificación de tercer nivel de estudios y 8 que han podido realizar estudios de cuarto nivel para beneficio de los estudiantes de esta unidad educativa. Existe solo un docente egresado de la especialidad de ingeniería mecánica que tiene la oportunidad de adquirir experiencia en el sector público.

Gráfico 4. Nivel de instrucción de los docentes

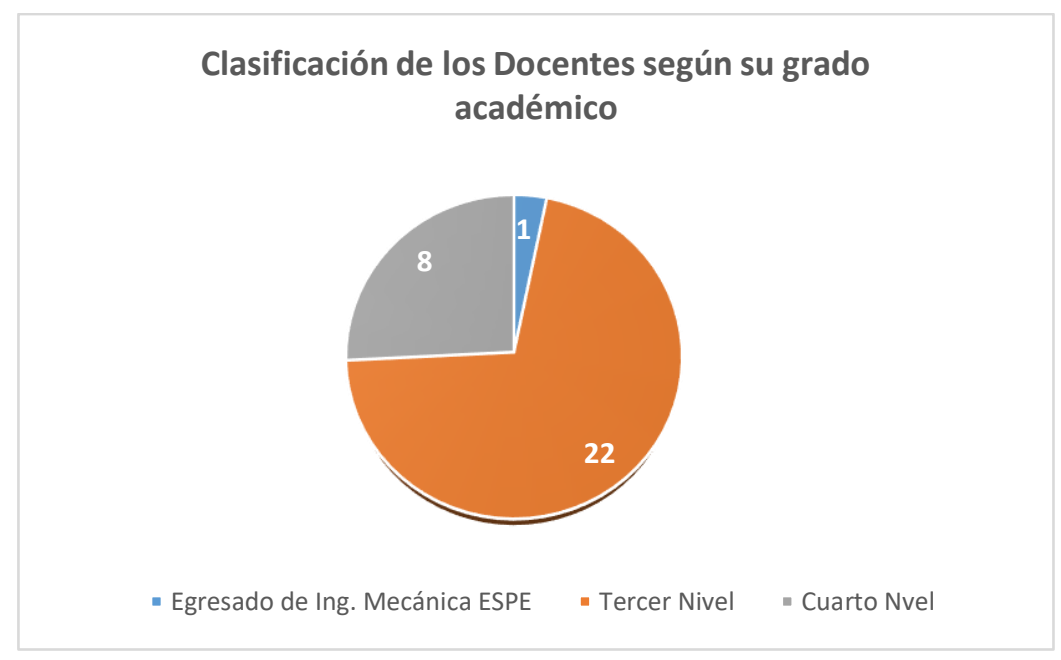

Fuente: Encuesta realizada por los autores

A los docentes citados, se los ha clasificado de acuerdo a su edad como se puede ver en el gráfico 5, y como se puede apreciar, la concentración de docentes mayoritariamente se encuentra en la edad comprendida entre los 31 y 40 años de edad.

Esto demuestra que la unidad educativa cuenta con un cuerpo docente joven para impartir sus conocimientos.

Gráfico 5. Docentes por rangos de edad

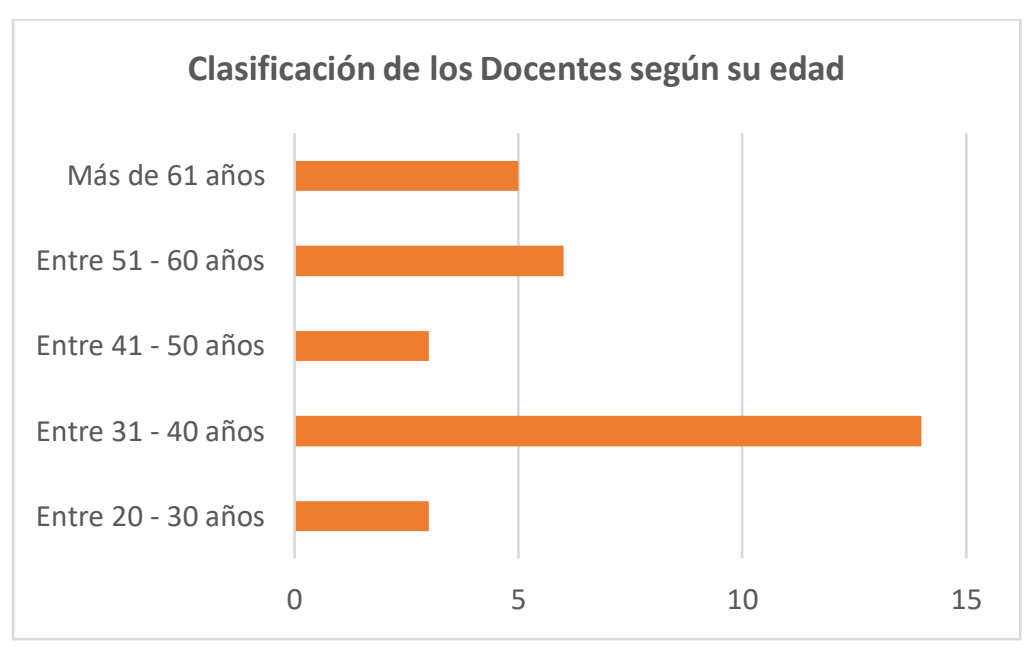

Fuente: Encuesta realizada por los autores

La primera pregunta de la encuesta fue sobre el grado de aprendizaje virtual, tanto padres de familia (PP1) como docentes (PD1) coinciden que los estudiantes han 
aprendido en un rango del $51 \%$ al $75 \%$ aunque existen opiniones importantes que señalan que un importante grupo de estudiantes han aprendido un porcentaje entre el $26 \%$ y el $50 \%$. Lo que significaría un retroceso en temas educativos en perjuicio de los estudiantes como se puede ver en el gráfico 6.

Gráfico 6. Aprendizaje de los estudiantes de la unidad educativa

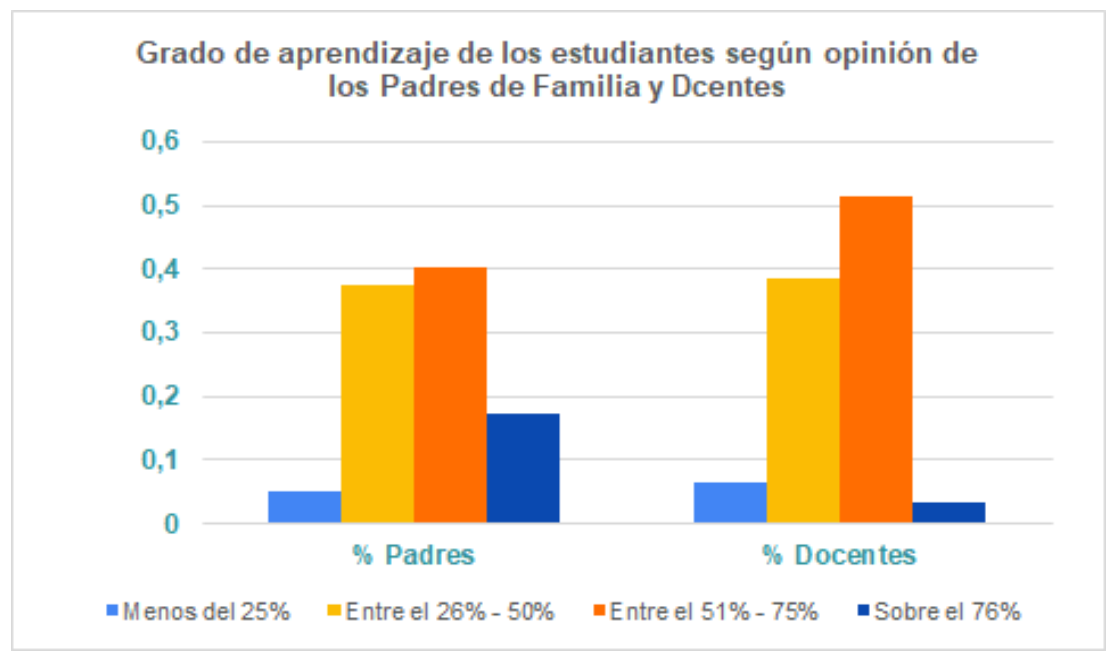

Fuente: Encuesta realizada por los autores

Un elemento importante se analiza en la segunda pregunta de la encuesta a los padres de familia (PP2) que fue acerca de su percepción sobre los docentes y su capacidad de llegar a los estudiantes, como se puede observar existe una importante opinión sobre la capacidad de los docentes para llevar su trabajo a cabo en pos del aprendizaje de sus hijos en casa.

El establecimiento temporal de la educación virtual en la educación general pública y privada por efectos de la pandemia, se puede sobrellevar de acuerdo a la opinión de los padres de familia de mejor forma con los docentes que cuenta el plantel educativo como se puede ver en el Gráfico 7.

Gráfico 7. Grado de comprensión de las clases de los docentes de la unidad educativa

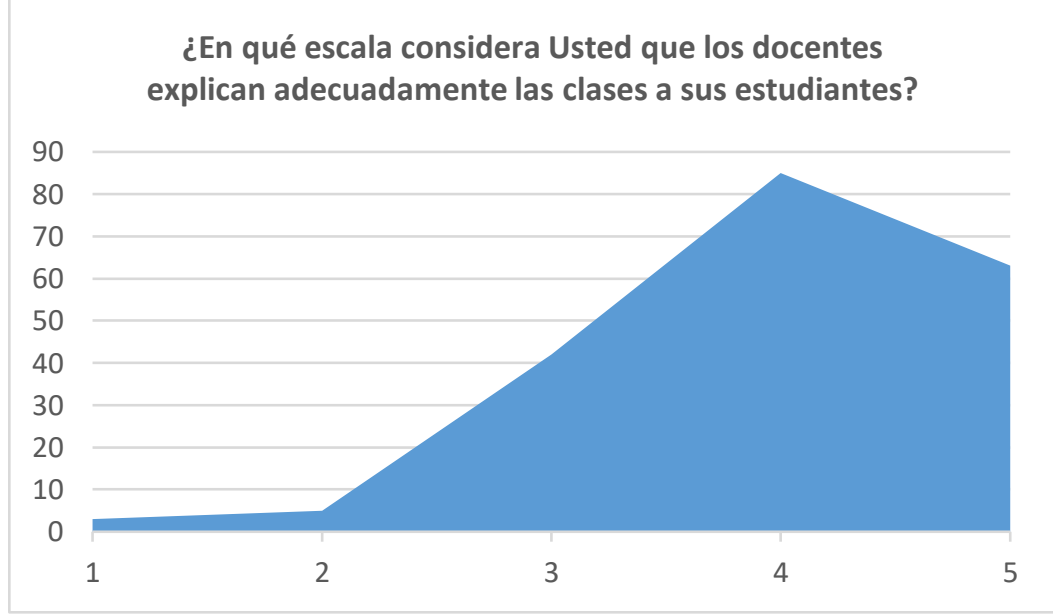

Fuente: Encuesta realizada por los autores 
En cuanto a la percepción del cuerpo docente en la pregunta 2 (PD2), la educación pública en el caso de la unidad educativa en investigación, si está preparada para asumir el reto de la educación virtual como se puede apreciar en el Gráfico 8:

Gráfico 8. El desafío de la educación virtual actualmente

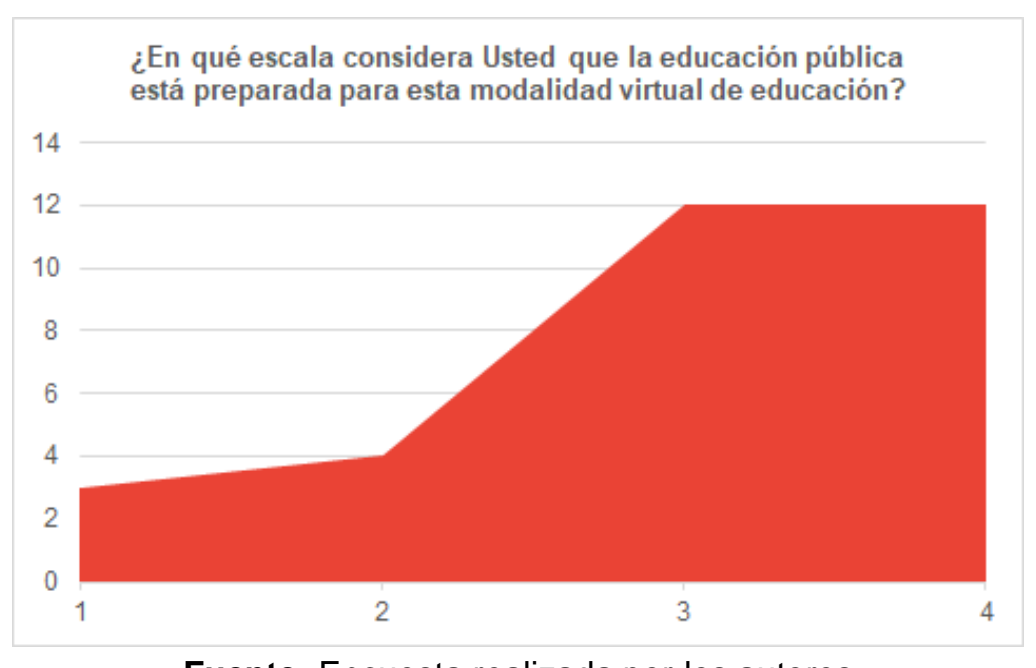

Fuente: Encuesta realizada por los autores

En la siguiente pregunta común formulada, la mayoría de padres de familia (PP3) y docentes (PD3) prefieren la educación presencial como mecanismo de aprendizaje tradicional y que hoy se mantiene.

Esto suena en cierta forma lógica si se profundiza en las razones que avalan lo afirmado como, por ejemplo: la concentración, el uso de equipos, la convivencia estudiantil, la interacción con docentes, etc, como se puede ver en el gráfico 9.

Gráfico 9. Preferencias de padres de familia y docentes por el tipo de educación

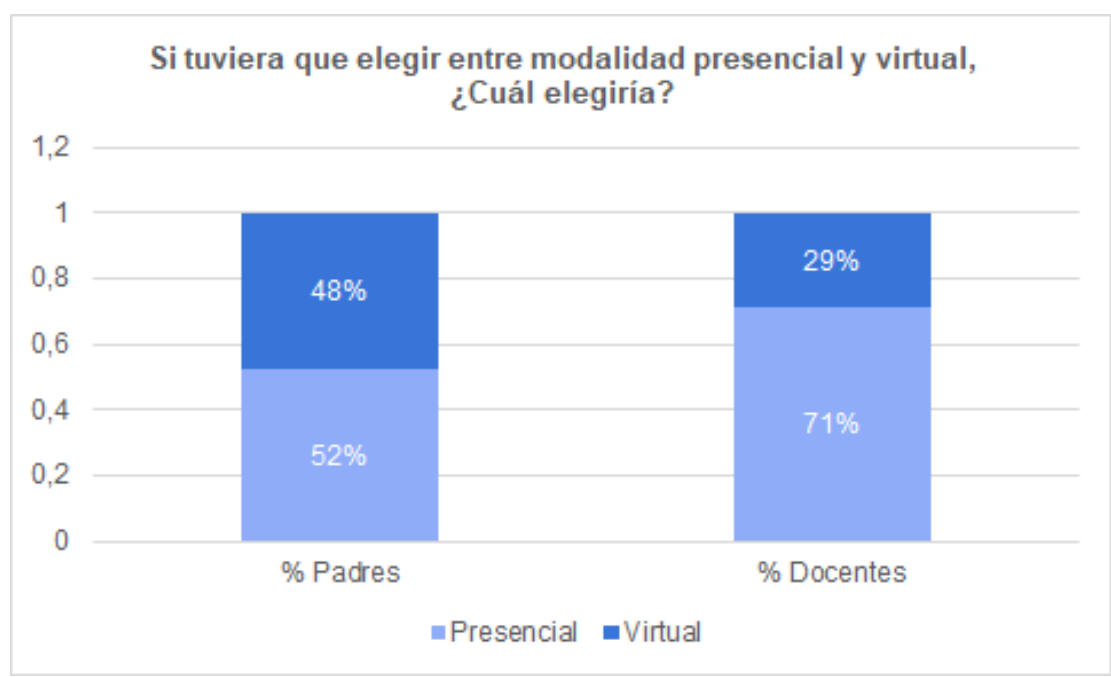

Fuente: Encuesta realizada por los autores 
La siguiente pregunta realizada a los padres de familia (PP4) fue sobre las tareas que sus hijos realizan en casa y que mayoritariamente 166 personas contestaron que, si son adecuadas para complementar el aprendizaje, mientras que 32 consideran que no han permitido el nexo con los estudiantes y en algunos casos no se han entendido las tareas, como se aprecia en el gráfico 10.

Gráfico 10. Percepción de las tareas que envían los docentes a los estudiantes

Las tareas que envía el docente al estudiante:

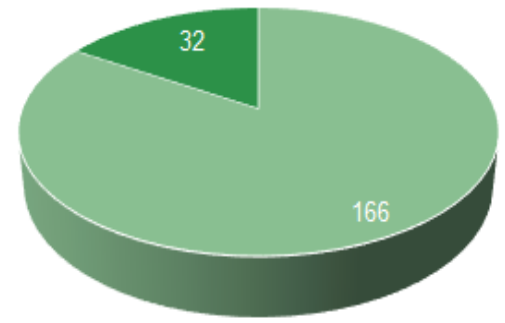

- Ayudan a complementar el aprendizaje

- No se entienden y consumen mucho tiempo

Fuente: Encuesta realizada por los autores

En tiempos de educación virtual, se les consultó a los padres de familia (PP5) con respecto a sus hijos y a los docentes (PD4) sobre los principales problemas que han experimentado.

Como se puede apreciar, los padres de familia en un $61 \%$ y los docentes en un $55 \%$ sostienen que el principal problema para la educación virtual es la mala conexión a internet seguido de la bulla en casa y que genera distracciones.

Cabe rescatar que un porcentaje importante de padres y docentes consideran que no existen dificultades y que se relaciona básicamente por los megas de internet contratados, así como también el sector donde residen y que son las causas que afectan el desarrollo normal de sus clases virtuales, como se puede ver en el gráfico 11 .

Gráfico 11. Problemas detectados por padres de familia y docentes para la educación virtual

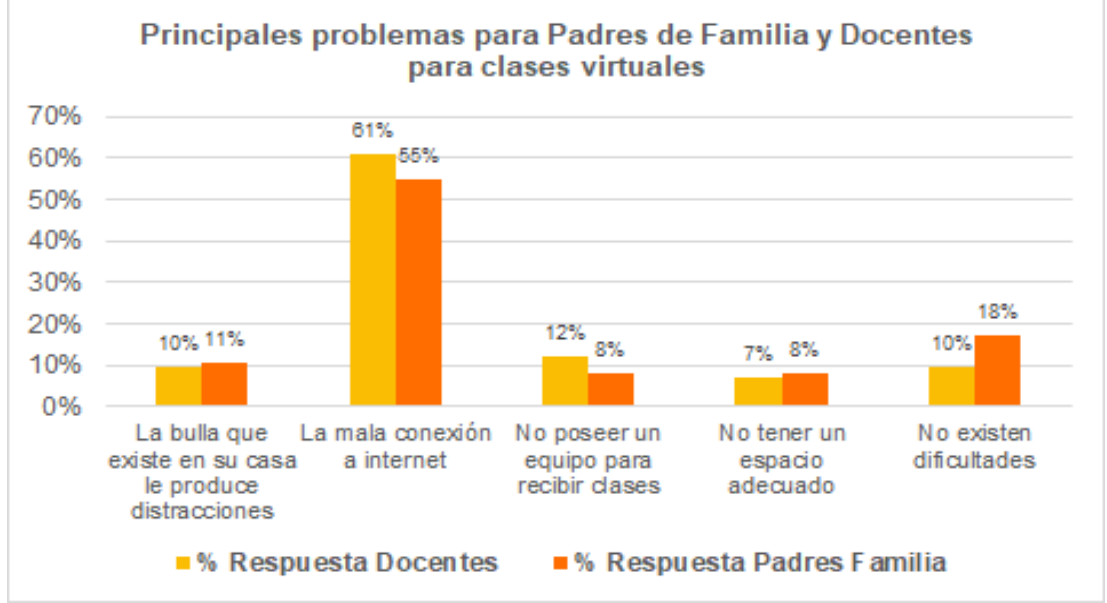

Fuente: Encuesta realizada por los autores 
Relacionado a la anterior pregunta, se les consultó a los docentes (PD5) sobre las plataformas que utilizan para dictar las clases en forma virtual, el 54\% utiliza microsoft teams y el $37 \%$ zoom como las plataformas más representativas y de aprendizaje para estudiantes y docentes para realizar sus videoconferencias en tiempo sincrónico, así se puede ver en el gráfico 12.

Gráfico 12. Uso de plataformas educativas por los docentes de la unidad educativa

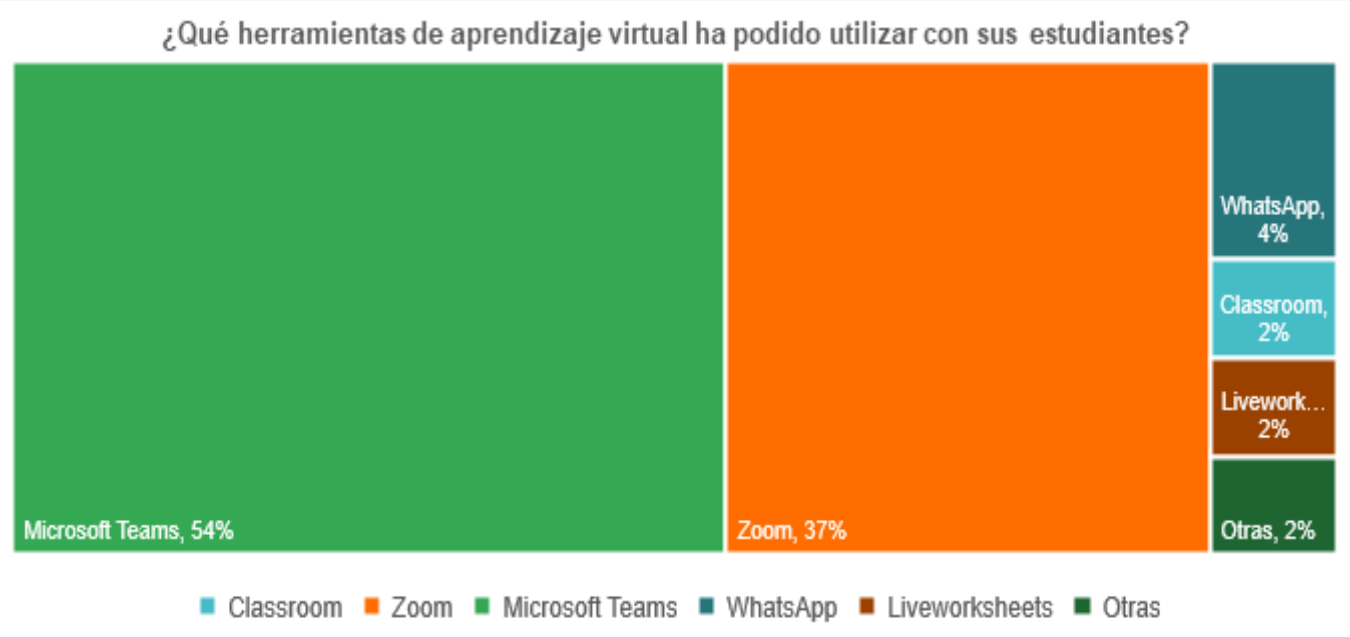

Fuente: Encuesta realizada por los autores

En cuanto a las herramientas para gamificar el aula virtual y conseguir un mejor aprendizaje se realizó la consulta a docentes (PD6), entre la variedad importante de las mismas y cuáles utilizan más y los resultados fueron: padlet con el $20 \%$, genially con el $16 \%$, kahoot, quizizz con el $13 \%$ y youtube con el $11 \%$.

Para esto ha sido necesario la capacitación en estas herramientas por parte de docentes y posteriormente de estudiantes. Esto se puede visualizar en el gráfico 13

Gráfico 13. Herramientas para gamificar el aula virtual por parte de los docentes

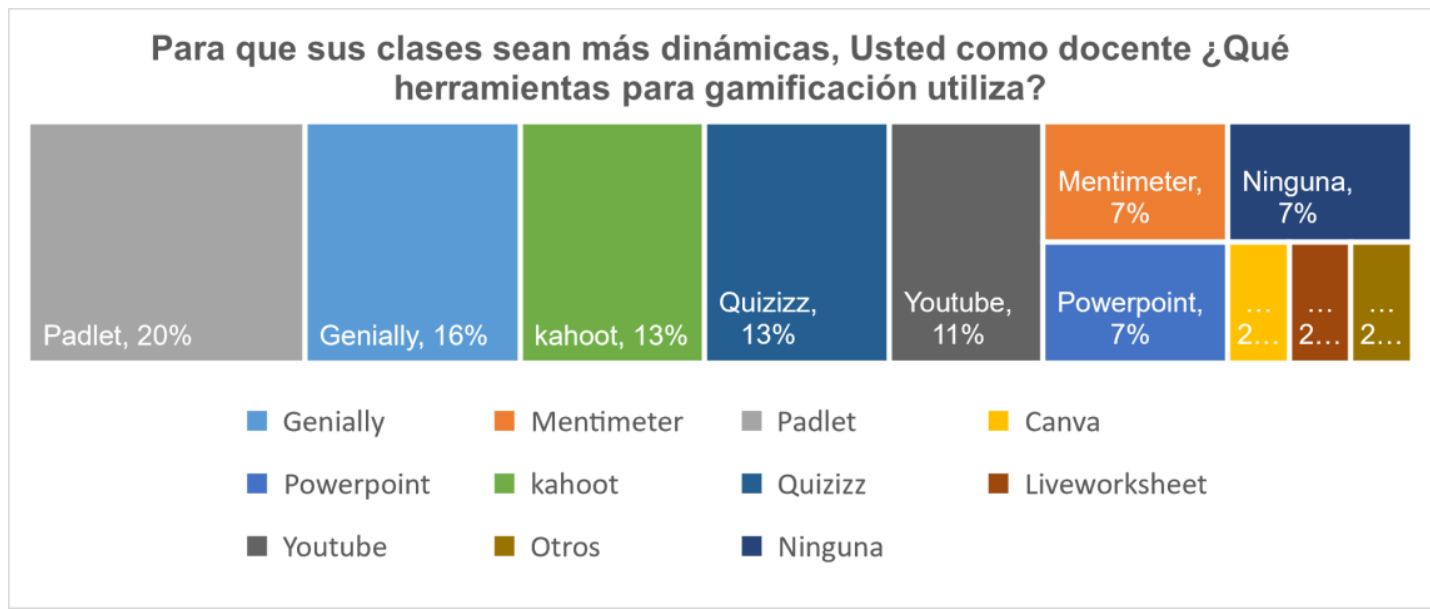

Fuente: Encuesta realizada por los autores

Para llevar a cabo este propósito en el uso de herramientas virtuales, se les consultó a los docentes (PD7) si poseen un equipo para dictar sus clases, obteniéndose un resultado 
del $100 \%$, sin embargo, vale la pena aclarar que muchos de estos equipos eran de tecnologías pasadas que limitaban el enlace con fluidez en las clases virtuales.

En el caso de inversiones a nivel económico, tanto padres de familia (PP6) como docentes (PD8) han tenido que efectuar desembolsos, que para el primer caso lo han hecho entre $\$ 101$ y $\$ 300$, mientras que, en el segundo grupo, su inversión fue de más de $\$ 500$ como se puede ver en el gráfico 14

Gráfico 14. Inversión en la compra de equipos para clases virtuales

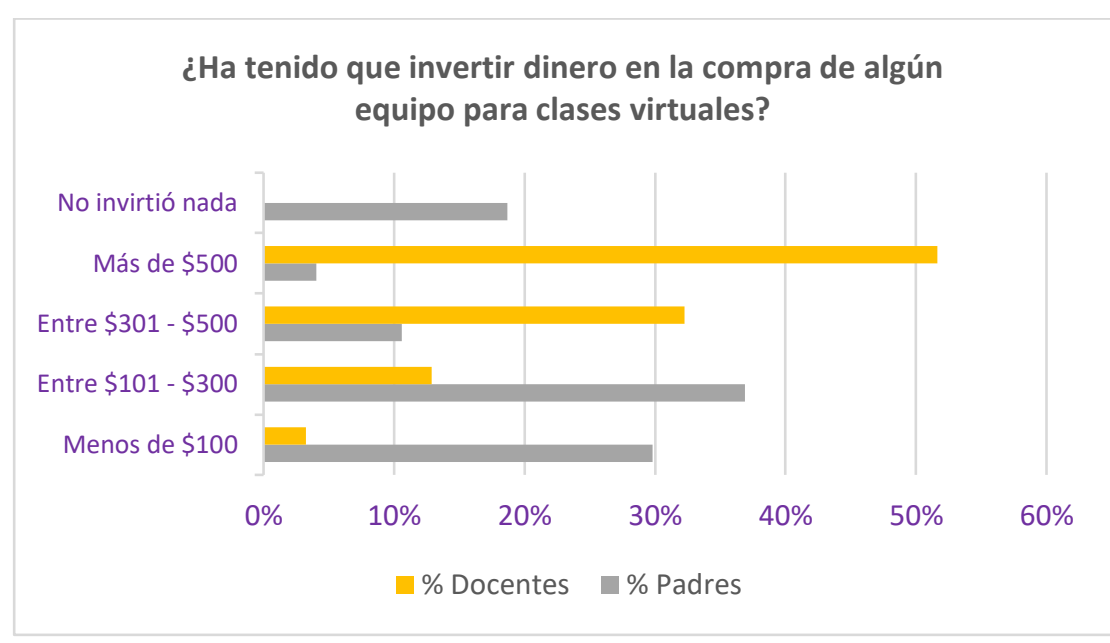

Fuente: Encuesta realizada por los autores

A los padres de familia (PP7) se les consultó acerca del impacto de la educación virtual en su presupuesto, obteniéndose las siguientes respuestas:

Gráfico 15. El gasto en las familias de los estudiantes de la unidad educativa

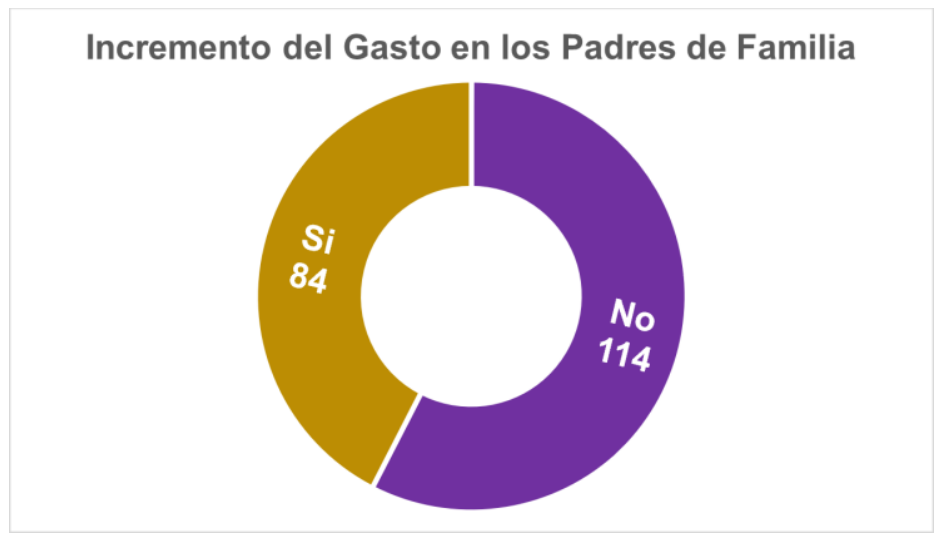

Fuente: Encuesta realizada por los autores

Según el gráfico 15, existe un importante grupo de familias, 84 de ellas que si se han visto impactadas por cuanto para recibir clases virtuales han debido invertir en equipos como: computadores, parlantes, cámaras, etc. 
En cuanto a los impactos a nivel económico y social, se les solicitó a los padres de familia (PP8) que evalúen entre 6 posibilidades, cuáles les han afectado a nivel hogar y de esa forma se ha construido el gráfico 16

Gráfico 16. Impacto económico - social por efectos de la pandemia

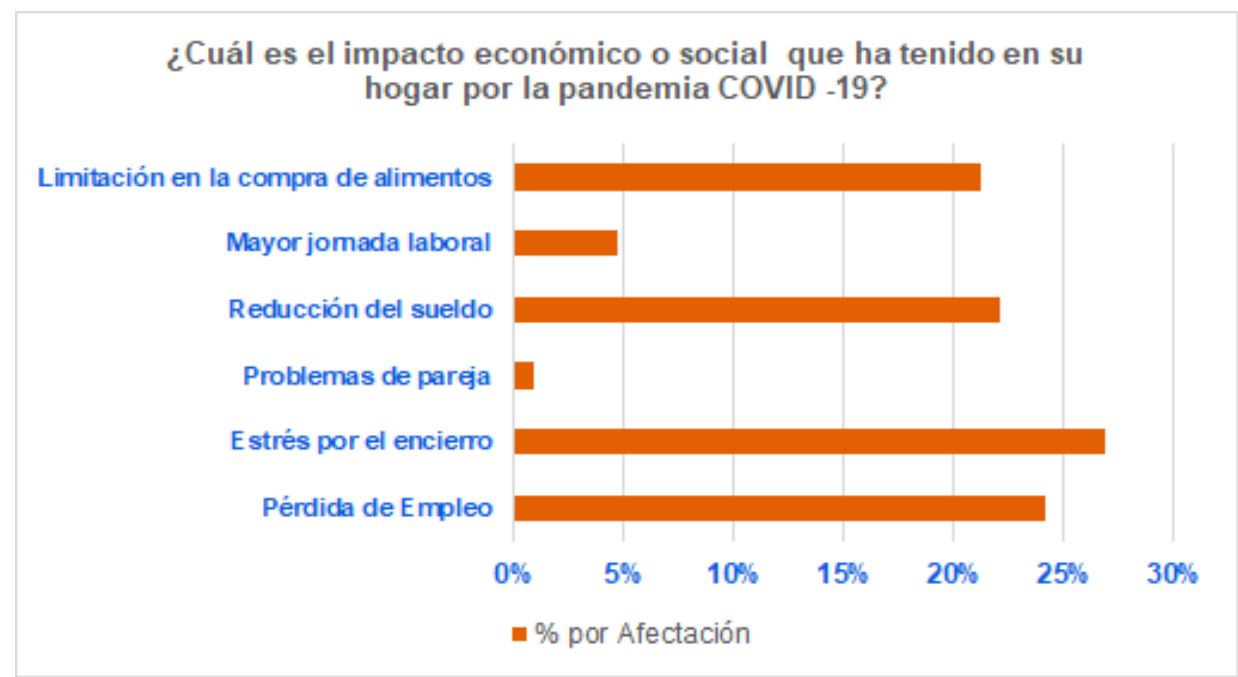

Fuente: Encuesta realizada por los autores

Como se puede apreciar, a nivel social el punto más vulnerable en las familias ha sido el estrés por el encierro que las autoridades del COE nacional y cantonal han determinado para frenar la ola de contagios por covid-19, sin embargo, los problemas de tipo sicológicos han aumentado. También se puede destacar que la pérdida de empleo ha afectado a las familias de la unidad educativa que estamos investigando, y que también es una característica a nivel nacional y mundial que golpea las economías domésticas.

En cuanto a los ingresos, se puede observar en el gráfico 17, que la mayoría de hogares (PP9) y docentes (PD9) de la unidad educativa, no tienen ingresos adicionales, en el primer caso 141 familias y en el segundo 27 docentes respectivamente.

Gráfico 17. Ingresos adicionales que perciben los Padres de Familia y Docentes

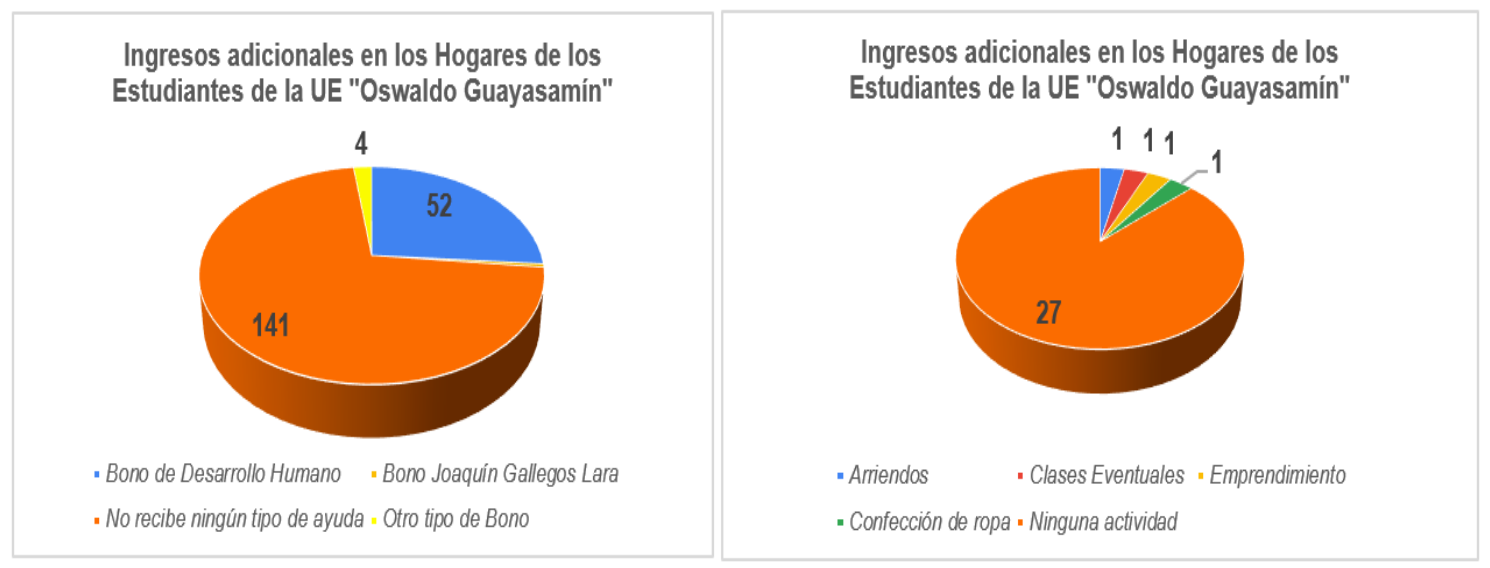

Fuente: Encuesta realizada por los autores 
Un aspecto importante a destacar es el ingreso que poseen las familias de los estudiantes (PP10) de la unidad educativa, debido a que un alto porcentaje percibe ingresos bajos, como lo puede notar el lector en el gráfico 18

Gráfico 18. Rangos de ingresos de los padres de familia

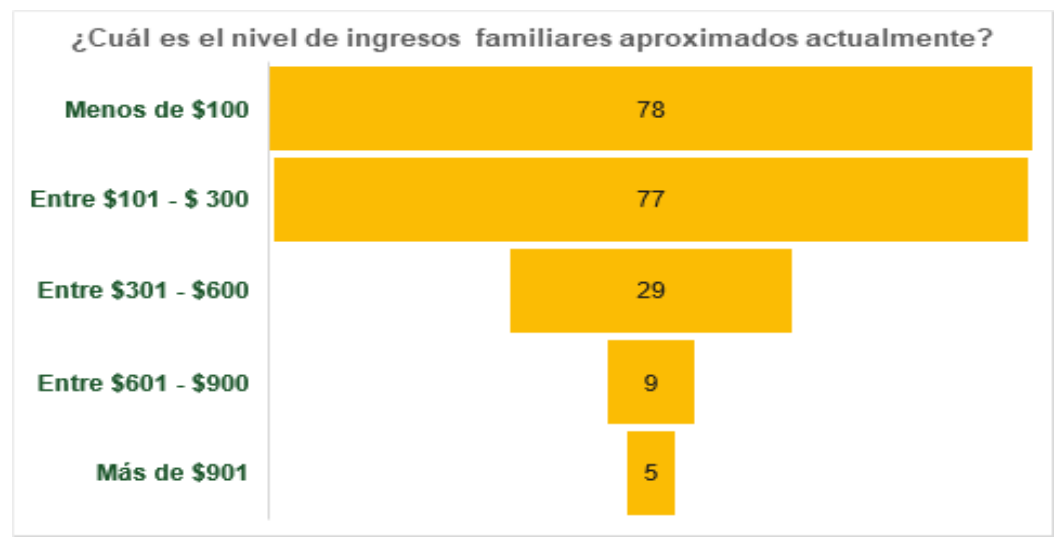

Fuente: Encuesta realizada por los autores

Es notorio que los hogares que se encuentran entre las dos primeras filas representan el $78 \%$ de la población y que viven por efectos de la pandemia con menos de $\$ 300$ al mes. Sin embargo, al consultarles a los padres de familia si durante el tiempo de pandemia pudieron generan alguna clase de emprendimiento (PP11), 131 hogares no lo hicieron; en tanto que 67 si lo hicieron producto de haber perdido sus trabajos o haber sufrido disminución en sus sueldos.

Un aspecto importante para los docentes (PD10) en el gráfico 19, fue la consulta en la encuesta relacionada al retraso en los pagos por parte del Gobierno Central, un 39\% ha acudido a endeudamientos en los bancos y cooperativas de la ciudad para suplir sus ingresos económicos, un $26 \%$ con sus tarjetas de crédito y un $22 \%$ con familiares que en cierta manera son quienes han ayudado a que los docentes puedan soportar el retraso del pago de sus sueldos.

Gráfico 19. Efectos del retraso en los pagos a los docentes por parte del gobierno central

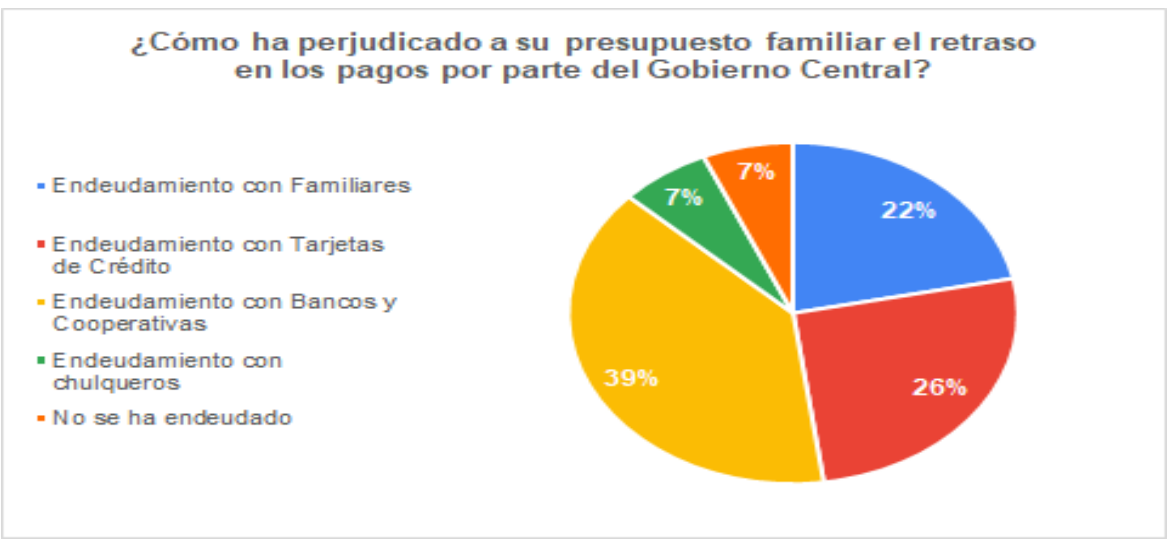

Fuente: Encuesta realizada por los autores 
Sin embargo, llama también la atención que un $7 \%$ de ellos han tenido que acudir a endeudamientos con chulqueros lo que significa problemas en las altas tasas de interés, así como también la incomodidad de posibles amenazas en los cobros.

Otro $7 \%$ no ha tenido la necesidad de endeudarse y mantiene cierto capital ahorrado para solventar estos momentos económicos por carencia de dinero en el gobierno para cumplir con sus obligaciones con los servidores públicos.

Resulta interesante analizar la compartición de espacios para recibir y dar clases virtuales para los hijos de los padres de familia (PP12) y los docentes (PD11) respectivamente. En el primer caso, el $85 \%$ de padres de familia considera que no existe ninguna afectación si se comparten los espacios con sus hijos, en tanto que los docentes consideran que si les afecta en su trabajo el poder compartir espacios que pueden generar inconvenientes con sus hijos, un $65 \%$ de ellos sienten esta problemática al impartir clases virtuales, el gráfico 20 muestra estos resultados.

Gráfico 20. Compartición de espacios para trabajo y clases virtuales en el hogar

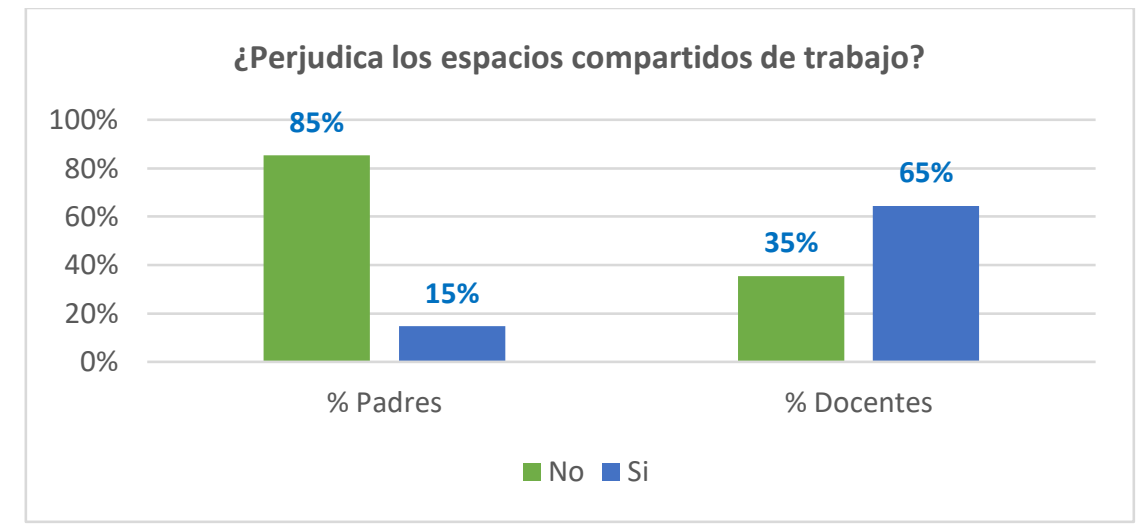

Fuente: Encuesta realizada por los autores

La siguiente pregunta compartida fue acerca del tiempo que podrían tanto padres de familia (PP13) como docentes (PD12) compartir con su hijo para el aprendizaje virtual, por lo que en el primer caso el $77 \%$ si dispone del tiempo para destinarlo a estar pendiente de sus hijos, en tanto que los docentes por su actividad, es complejo que puedan compartir tiempos, por lo que un $52 \%$ no dispone de tiempo para realizar el seguimiento en casa a sus propios hijos, así se puede apreciar en el gráfico 21

Gráfico 21. Tiempo para destinarlo a los hijos

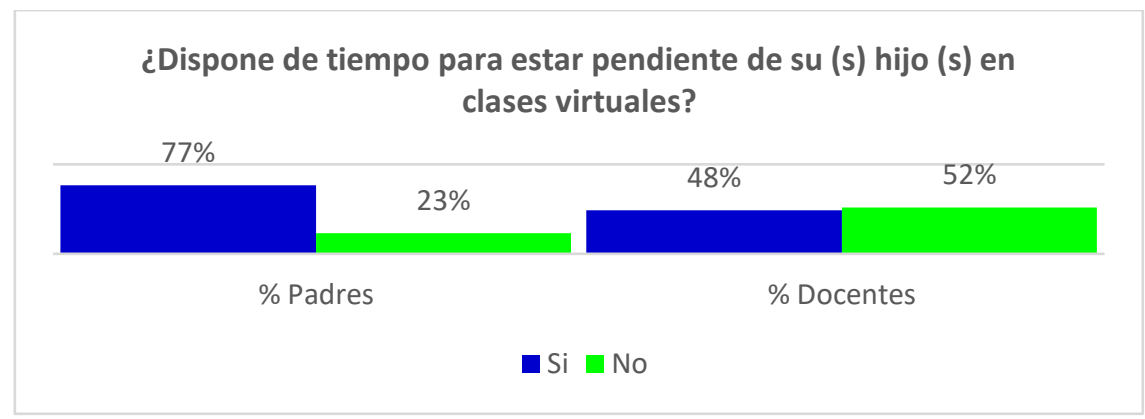

Fuente: Encuesta realizada por los autores 
Se les consultó a los padres de familia acerca de los cambios que han notado en sus hijos (PP14) y también en la segunda encuesta a los docentes (PD13) por la educación virtual.

En el primer caso, el 34\% del total encuestado sostiene que el estrés fue su principal problema y se complementa con la pregunta donde todo el hogar padece de lo mismo, luego el aburrimiento en casa con el $31 \%$ como los más representativos.

En cuanto a los cambios en los docentes, de forma similar el estrés con el $31 \%$ es el aspecto negativo que más les afectó, seguido de la mayor carga laboral experimentada con el $28 \%$ por los horarios, tareas, preparación de plataformas, etc. Esto se puede ver en el gráfico 22.

Gráfico 22. Cambios experimentados en estudiantes y docentes por causa del confinamiento y distanciamiento social

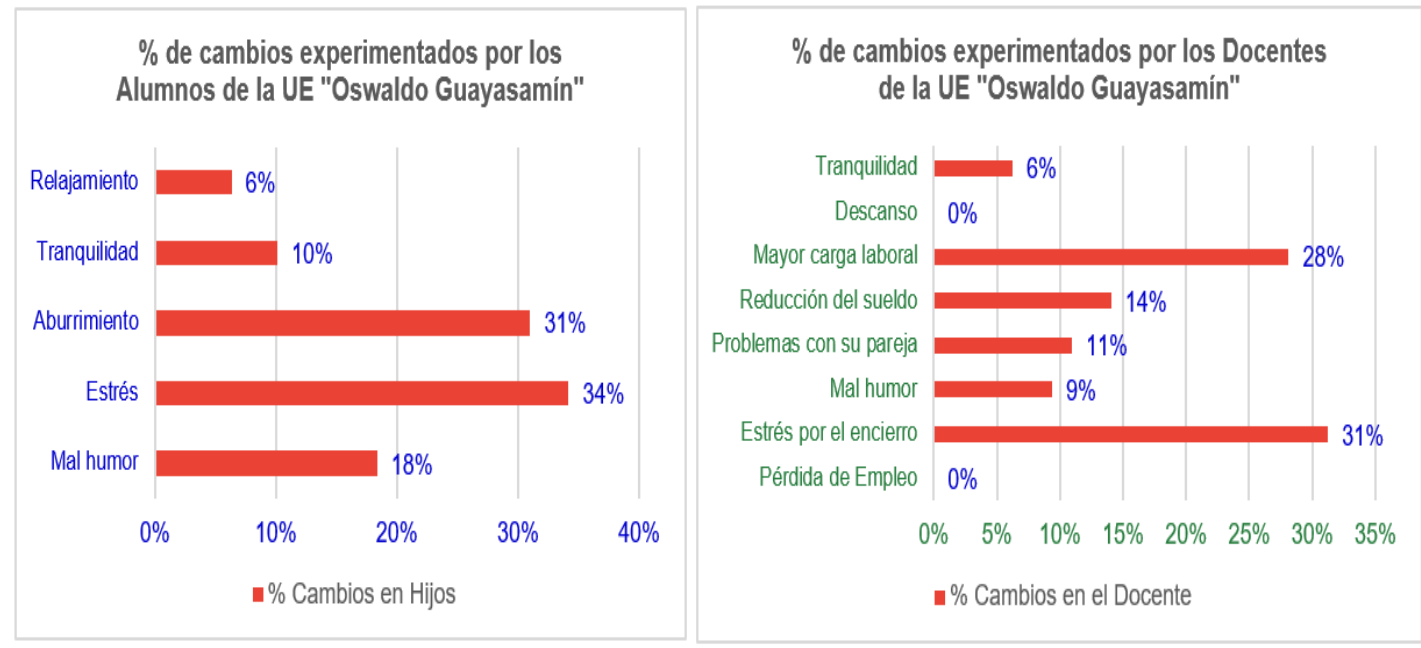

Fuente: Encuesta realizada por los autores

Un último aspecto que se analizó es lo relacionado a las actividades complementarias que realizan tanto estudiantes (PP15) como docentes (PD14), rescatándose en el primer caso que el 39\% de estudiantes realizan tareas de casa apoyando en el hogar, luego un $29 \%$ utiliza el tiempo en el uso del celular y dispositivos, pero para actividades de recreación y juegos y apenas pocos para investigar, un $22 \%$ del total dedica su tiempo a hacer deporte mientras los horarios del COE nacional lo permiten.

Ahora bien, en el caso de los docentes, el 27\% dedica su tiempo a preparar clases de los próximos días, un $24 \%$ comparte los espacios y tareas del hogar con su esposa e hijos, en tanto que un $22 \%$ se auto-capacita en youtube y redes sociales acerca de aspectos relacionados a su especialización y otras actividades de aprendizaje, como se puede ver en el gráfico 23. 
Gráfico 23. Actividades complementarias de estudiantes y docentes de la unidad educativa

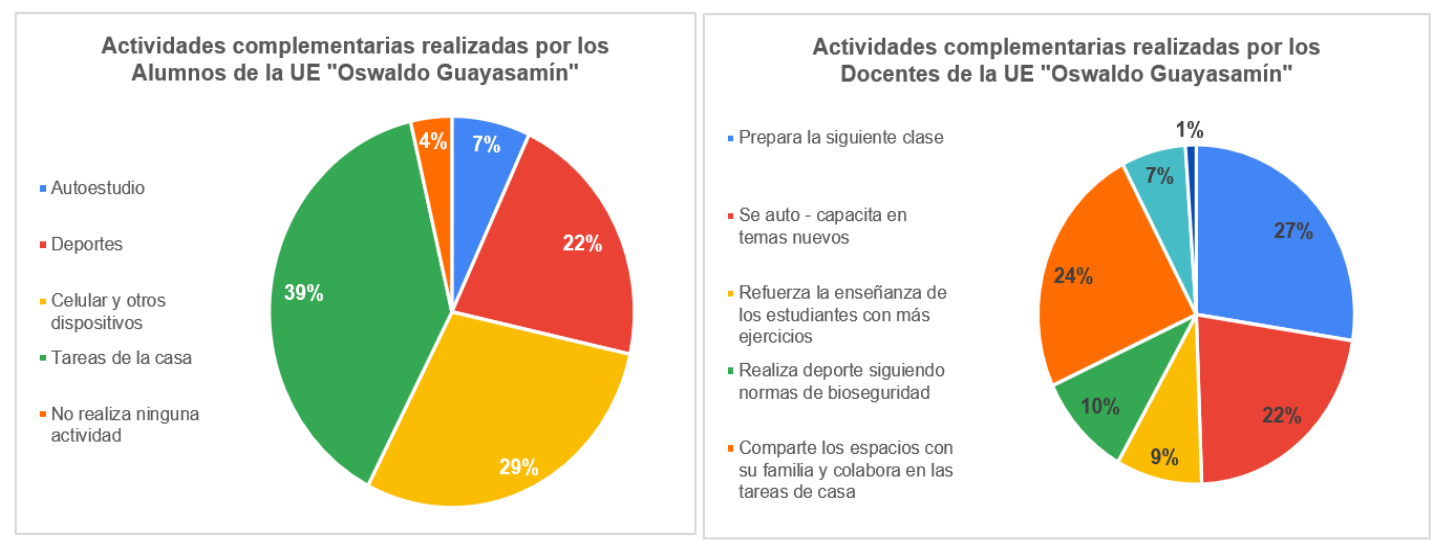

Fuente: Encuesta realizada por los autores

\section{Aspectos a considerar}

El problema de la pandemia a nivel mundial ha generado graves problemas en el tema educativo, así (Herberth Alexander, 2020) asume una posición crítica en cuanto a la convivencia social y sobre la preparación de las instituciones educativas y las familias para asumir este reto importante de educarse y aceptar los desafíos de compartir las actividades en familia, señalando que, para eso también hay que educarse.

Estas circunstancias se las analiza bajo el enfoque del estrés, el cual necesita ser canalizado para evitar rupturas sociales mientras la vacuna del COVID-19 aún no esté disponible para la mayor parte de la población, entonces la comunidad estudiantil no puede volver a la educación presencial porque se podría generar un grave problema de contagios masivos. En todo caso estaremos sujetos a las decisiones de las autoridades educativas.

En términos económicos, "Ecuador se contrajo un 7,8\% en el 2020 por efectos de la pandemia del coronavirus, que golpeó con fuerza el consumo de hogares y del gobierno, la inversión y las exportaciones" (Reuters, 2021).

En esta línea el Fondo Monetario Internacional (FMI) señala que Ecuador al año 2021, será la penúltima economía en crecer después de Venezuela que experimentará otra contracción anual, con el $2.5 \%$ y $-10 \%$ respectivamente. (Ekos, 2021), lo cual se traduce en menos recursos destinados al gasto social, concretamente a educación.

En este sentido, el núcleo familiar fue afectado por la pandemia y el confinamiento, el docente ha tenido que adaptarse a estos entornos virtuales que antes posiblemente solo los veía superficialmente, sobre todo los docentes que llevan años bajo la enseñanza tradicional.

Como bien menciona, esto causa incertidumbre acerca del uso adecuado de las TIC's, claro se establecen planes de contingencia por parte de los Ministerios de Educación de 
cada país, particularmente el caso de Paraguay se menciona la utilización de entornos como Google Classroom, Zoom, Teams, entre otros. (Britez, 2020)

La pandemia permitió migrar de la presencialidad a la virtualidad, desarrollando en Estudiantes y Docentes habilidades didácticas relacionadas a aspectos pedagógicos que como requisito fundamental tiene el conocimiento de la tecnología. (Carrasco Cosa, Caicedo Merizalde, Savedra Valdiviezo, \& Ochoa Sangurima, 2021).

Este fue un primer tropiezo para los docentes que en gran parte no entendían las herramientas virtuales al punto que algunos pensaron como opción la jubilación. "En el caso de Ecuador, el acceso a internet fijo ha aumentado durante los últimos años, alcanzando al 37.2\% de los hogares en 2018. Sin embargo, la persistente brecha digital podría penalizar especialmente a los individuos más pobres o que viven en zonas rurales en su acceso al teletrabajo y la educación online. Solamente el 16,1\% de los hogares en áreas rurales tenían acceso a internet en 2018, frente al 46.6\% en áreas urbanas (INEC, 2018a). Además, solamente el 39.9\% de los individuos en hogares pertenecientes al $20 \%$ más pobre usaban internet en 2017 , frente a $73.7 \%$ en hogares más ricos" (OCDE, 2020)

Similar situación se puede observar en México, donde existe al igual que Ecuador diversidad de culturas que generan condicionamientos en la educación sobre todo en los más pobres y los más alejados de las ciudades donde al parecer el confinamiento ha cambiado el comportamiento de la población y ha requerido la adquisición de equipos en unos casos, lo que ha deteriorado las condiciones económicas sobre todo de familias de escasos recursos.

Por otro lado, el docente ha tenido una condición a manera de revalorización en su capacidad para educar a distancia lo que ha ocasionado diferenciaciones notorias en la calidad de la educación, lo que conlleva a que las autoridades gubernamentales deban replantear los contenidos académicos encaminados a mejorar el aprendizaje sobre todo de los más vulnerables e históricamente excluidos. (Plascencia González, 2020)

Un estudio realizado por Ramírez Ortiz, Castro Quintero, Lerma Córdoba, Yela Ceballos , y Escobar Córdoba (2020) señalan que las alteraciones psicológicas por efectos del confinamiento en el hogar son múltiples, sobre todo el estrés que puede llegar a provocar la interrupción del sueño, y si esto se aplica a estudiantes, padres de familia y docentes, la situación sería preocupante a nivel social, como se manifiesta en el estudio realizado en la unidad educativa objeto de esta investigación.

Hay que considerar un estudio realizado en España donde se consideran importantes elementos que abonan a este estudio. Por una parte, se señala que la pandemia desde su aparecimiento masivo en el año 2020, ha sido un escenario sin precedentes que ha ocasionado que el docente se adapte al teletrabajo mientras que el alumno por el confinamiento ha debido generar una especie de nueva adaptación. 
Se añade que se ha dividido el trabajo docente - estudiante en enfoques sincrónicos y asincrónicos de trabajo y donde en el punto central están las familias como guías de educación y complemento del docente. Complementa el análisis enfocándose en el tipo de familia que tiene el estudiante para que su formación sea adecuada; sin embargo, existen problemas que se deben tener presentes y es que en varias familias existen turnos para uso de las computadoras.

La mala conexión de internet, el acompañamiento de los padres en las clases con sus hijos, así como los problemas económicos en el interior de las familias ante los actuales problemas laborales, estas realidades se presentan en varios hogares no solo de estudiantes sino también de los docentes. (Muñoz Moreno \& Lluch Molins, 2020).

Un impacto importante analizado por la OCDE (2020), ha sido el cierre de las escuelas ${ }^{8}$ por el COVID - 19 y que presionan a la desigualdad entre grupos económicos donde la brecha será más grande por las tecnologías en uso para la virtualidad y también por la responsabilidad de los padres en el acompañamiento y aprendizaje de sus hijos.

Sobre este último punto, el $14.8 \%$ de estudiantes de primaria en Ecuador tienen computador con internet en condiciones de pobreza mientras que de los hogares con mayores ingresos el 53.6\% disponen estas herramientas según cifras al 2018. Pensando en esto, el ministerio de educación del Ecuador, ha implementado en el país modalidades por radio en algo más de 1000 radios rurales comunitarias y 160 canales de televisión ${ }^{9}$ para el acceso al conocimiento.

El impacto en la educación es uno de los vértices sobre los cuales la pandemia ha dejado huellas, razón por la cual el ministerio de educación ha puesto en marcha desde el año 2020 algunos planes para mitigar estos efectos como se citan a continuación:

$\checkmark$ Plan educativo "aprendemos juntos en casa": acompañamiento a estudiantes y docentes en temas pedagógicos mediante un portal educativo.

$\checkmark$ Atención educativa a través de medios no convencionales: apoyo a comunidades $\mathrm{y}$ pueblos que no tienen conectividad o es mala mediante el canal digital "EDUCA" entre otras.

$\checkmark$ Acceso a recursos digitales: se habilitaron cuentas de classroom y microsoft teams a estudiantes para acceso a clases.

$\checkmark$ Capacitación docente: guías y evaluaciones docentes para la modalidad virtual. OCDE (2020)

Complementando lo anterior, para García (2020), las clases consideradas a distancia pueden tener varios distractores al realizarse desde el hogar como: el celular, desayuno,

\footnotetext{
${ }^{8}$ De acuerdo a la UNICEF, "El cierre de las instalaciones educativas ha afectado aproximadamente a 4.4 millones de estudiantes2. Los niños, niñas y adolescentes en mayor situación de vulnerabilidad -con discapacidad, en situación de pobreza, refugiados y migrantes, y las niñas en particular- enfrentan mayores barreras en el acceso a la educación" (UNICEF, 2021).

${ }^{9}$ El Ministerio de Educación en su página web ha habilitado para el docente y estudiante plataformas importantes para la inclusión educativa. Ver: https://recursos2.educacion.gob.ec/
} 
televisor, etc. Ahora bien, en las videoconferencias las dificultades se relacionan a la debilidad de la señal, problemas de audio o video, retrasos en el video, así como no disponer de espacios adecuados para evitar ruidos o distractores que no le permitan al estudiante o al docente a desarrollar las clases virtuales.

En el caso de instituciones educativas de Mendoza en Argentina, la herramienta más utilizada para la comunicación entre estudiantes y docentes ha sido WhatsApp. (Expósito \& Marsollier, 2020). En este sentido Britez (2020) señala que en Paraguay y Argentina se utilizaron entre otros al WhatsApp y al Classroom aún cuando muchos docentes no estaban familiarizados.

Se encontraron problemas relacionados a que los padres no podían acompañar a sus hijos en las clases virtuales, problemas para manejar el celular en unos casos, dificultades económicas para cancelar las pensiones, entre otros. Sin embargo preocupa a Britez que se pueda producir una suerte de desgaste para los estudiantes, los padres y docentes en especial y conlleve efectos más graves si no se prepara alguna estrategia a nivel educativo y también que existen asignaturas que no se pueden manejar en forma virtual como si en forma presencial y en este punto los autores de este trabajo, han identificado sobre todo a las carreras técnicas como: mecánica, electricidad, carpintería, etc., así como las que son de acondicionamiento físico o deportivo en el caso de las competencias de las selecciones colegiales.

\section{Conclusiones}

- La pandemia ha generado problemas en la economía de los hogares provocando crisis en algunos casos donde se ha llegado al límite de no poder satisfacer ciertas necesidades producto del presupuesto familiar limitado. El $93 \%$ de docentes fueron afectados económicamente por el retraso de sus sueldos mensuales por parte del gobierno central, de las cuales el $39 \%$ ha tenido que acudir a un endeudamiento extraordinario para suplir la necesidad de la educación virtual a través de créditos en instituciones financieras, el uso de sus propias tarjetas de crédito y la ayuda de familiares para solventar las necesidades de sus hogares.

- En este enfoque, el impacto social en los estudiantes resulta preocupante, se señala que el $34 \%$ han tenido un grado de estrés importante, deteriorando así su salud continuamente, el $31 \%$ manifestó que sufre de aburrimiento en casa. A nivel docente, se señala que la compartición de la intimidad de su hogar con sus alumnos ha generado cierto problema, el $65 \%$ de docentes encuestados señalan que han tenido que acondicionar un espacio en su hogar y asumir los costos de energía eléctrica producto de su actividad educativa y lo que preocupa es que un $31 \%$ de ellos han sufrido estrés por el encierro al otro lado de la pantalla, afectando incluso sus relaciones familiares, así lo señala el $11 \%$ de los docentes.

- La pandemia ha generado una nueva forma de educación tanto para las familias como para los docentes y se pone de manifiesto la capacidad de adaptación a las TIC's mediante el uso de las plataformas de enseñanza como teams 54\% y zoom $37 \%$ como las más representativas y el acondicionamiento de las aulas virtuales para 
generar procesos de enseñanza- aprendizaje adecuados que motiven a los estudiantes estar pendientes de sus clases aún cuando los horarios en unos casos son demasiado extensos y provocan el efecto contrario al aprendizaje. Para fortalecer el aprendizaje, el docente ha tenido que gamificar su aula virtual con plataformas como padlet $20 \%$, genially con el $16 \%$ y quizziz con el $13 \%$.

- Finalmente, es adecuado considerar cómo se aprovecha el tiempo extra clase virtual, donde el 39\% de los estudiantes realizan tareas de casa y el 29\% de ellos pasan en el celular en juegos de adicción. Para el caso de los docentes, el 27\% dedica su tiempo libre a preparar clases y el $24 \%$ apoya a su familia en las tareas del hogar.

\section{Referencias bibliográficas}

Asamblea Nacional del Ecuador. (2018). Constitución de la República del Ecuador. Ecuador. Recuperado el 28 de 05 de 2021

Britez, M. (2020). La educación ante el avance del COVID-19 en Paraguay. Comparativo con países de la Triple Frontera. Scielo, 1 - 15. Obtenido de https://preprints.scielo.org/index.php/scielo/preprint/view/22/579

Carrasco Cosa, O., Caicedo Merizalde, J. G., Savedra Valdiviezo, O. A., \& Ochoa Sangurima, V. L. (2021). Fundamentos Pedagógicos para la enseñanzaaprendizaje de la Educación Física en Modalidad Virtual: Un reto actual. Ciencia Digital, 5(1), $232 \quad$ - 251. Obtenido de https://cienciadigital.org/revistacienciadigital2/index.php/CienciaDigital/article/ view/1542/3905

Castillo, M. (2010). La profesión docente. Scielo, 902 - 907 . Obtenido de https://scielo.conicyt.cl/pdf/rmc/v138n7/art17.pdf

Ecured. (s.f.). Estudiante. Obtenido de https://www.ecured.cu/Estudiante

Ekos. (25 de 05 de 2021). Crecimiento del PIB 2021 en Latinoamérica. Obtenido de https://ekosnegocios.com/articulo/crecimiento-del-pib-2021-en-latinoamerica

Expósito, C. D., \& Marsollier, R. G. (2020). Educación y Humanismo. Educación y Humanismo, 22(39), $1 \quad$ - $22 . \quad$ Obtenido de http://revistas.unisimon.edu.co/index.php/educacion/article/view/4214/4771

García García, M. D. (2020). La docencia desde el hogar. Una alternativa necesaria en tiempos del Covid 19. Polo del Conocimiento, 5(4), 304 - 324. Obtenido de https://dialnet.unirioja.es/servlet/articulo?codigo=7398376

Gómez, V. L., \& Álvarez, G. (2020). Tecnologías digitales en la escuela primaria: las perspectivas de los docentes sobre su inclusión y la enseñanza en las aulas. Virtualidad, educación y ciencia(20), 9-26. 
Herberth Alexander, O. (2020). La educación en tiempos de pandemia: visión desde la educación superior. Disruptiva. doi:2706-5421

Inca Ruiz, G. P., \& Inca León, A. C. (2020). Evolución de la enfermedad por coronavirus (COVID-19) en Ecuador. La Ciencia al Servicio de la Salud y la Nutrición, $\quad 11(1), \quad 5 \quad-15 . \quad$ Obtenido de http://revistas.espoch.edu.ec/index.php/cssn/article/view/441/422

Instituto Nacional de Evaluación Educativa. (2018). La educación en Ecuador: logros alcanzados y nuevos desafíos. Obtenido de https://www.evaluacion.gob.ec/wpcontent/uploads/downloads/2019/02/CIE_ResultadosEducativos18_20190109.p df

Jama Zambrano, V., \& Cornejo Zambrano, J. (2016). Las condiciones socioeconómicas y su influencia en el aprendizaje: un estudio de caso. Revista científica Dominio de las Ciencias, II(1), 102-117.

Ministerio de Economía y Finanzas. (2020). Informe Anual de Ejecución. Obtenido de https://www.finanzas.gob.ec/wpcontent/uploads/downloads/2020/04/Inf_ejec.presupuestaria_2019.pdf

Ministerio de Educación del Ecuador. (2013). Ecuador: indicadores educativos 2011 2012 Obtenido de https://educacion.gob.ec/wpcontent/uploads/downloads/2013/10/Indicadores_Educativos_102013_DNAIE.pdf

Mujica, R. (2020). Ecosistema Tecnológico: Como herramienta transformadora para el proceso de aprendizaje significativo. Revista cientifica Aula Virtual, 1(1), 6-14.

Muñoz Moreno, J., \& Lluch Molins, L. (2020). Consecuencias del Cierre de Escuelas por el Covid-19 en las Desigualdades Educativas. Revista Internacional de Educación para la Justicia Social, 9(3), 2 - 17. Obtenido de https://revistas.uam.es/riejs/article/view/12216/12089

OCDE. (2020). Impacto Social del Covid - 19 en Ecuador: desafíos y respuestas. 4. Obtenido de https://www.oecd.org/dev/Impacto-social-COVID-19-Ecuador.pdf

Plascencia González, M. (2020). La tele-educación como un desafío en una sociedad con alta desigualdad social: el caso de las infancias indígenas y rurales. Sinergias Educativas, 5(4), 101 - $118 . \quad$ Obtenido de http://sinergiaseducativas.mx/index.php/revista/

Primicias. (11 de 2020). Reducción del presupuesto del Estado en 2020 llega al 8,3\%. Obtenido de https://www.primicias.ec/noticias/economia/salud-educacionreduccion-presupuesto/ 
Ramírez Ortiz, J., Castro Quintero, D., Lerma Córdoba, C., Yela Ceballos , F., \& Escobar Córdoba, F. (2020). Consecuencias de la pandemia de la COVID-19 en la salud mental asociadas al aislamiento social. Colombian Journal of Anesthesiology, $\quad 48(4), \quad 1 \quad-4.48$ Obtenido de http://www.scielo.org.co/pdf/rca/v48n4/es_2256-2087-rca-48-04-e301.pdf

Razeto, A. (2016). El involucramiento de las familias en la educación de los niños. Cuatro reflexiones para fortalecer la relación entre familias y escuelas. Páginas de Educación, 9(2), $190 \quad$ - $216 . \quad$ Obtenido de https://dialnet.unirioja.es/servlet/articulo?codigo $=6761491$

Reuters. (01 de 04 de 2021). América Economía. Obtenido de https://www.americaeconomia.com/economia-mercados/finanzas/economia-deecuador-se-contrae-un-78-en-el-2020-por-efectos-de-la

Scola, A. (2012). Familia y Sociedad. Humanitas, 1 - 28. Obtenido de https://espanol.clonline.org/cm-files/2012/09/04/familia-.pdf

Suárez Huz, Y. (2018). Impacto de la educación virtual y las TIC en la andragogía. Revista Ciencias de la Educación, 28(51), 1316-5917.

UNICEF. (14 de 01 de 2021). Información sobre el Coronavirus (COVID-19). Obtenido de https://www.unicef.org/ecuador/comunicados-prensa/priorizar-laeducaci\%C3\%B3n-para-todos-los-ni\%C3\%B1os-y-ni\%C3\%B1as-es-el-caminola-recuperaci\% $3 \% \mathrm{~B} 3 \mathrm{n}$

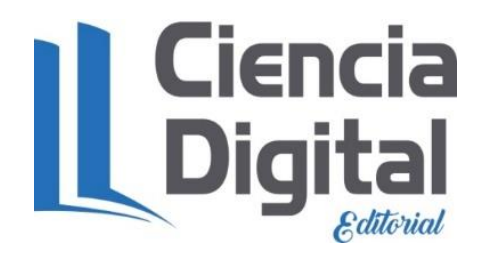




\section{PARA CITAR EL ARTÍCULO INDEXADO.}

Altamirano P, H. R., Cadena P, V. J., \& Arias V, B. E. (2021). Educación virtual y su impacto socio - económico en los estudiantes y docentes de una unidad educativa. Explorador Digital, 5(3), 85-109. https://doi.org/10.33262/exploradordigital.v5i3.1771

\section{Liencia}

El artículo que se publica es de exclusiva responsabilidad de los autores y no necesariamente reflejan el pensamiento de la Revista Explorador Digital.

El artículo queda en propiedad de la revista y, por tanto, su publicación parcial y/o total en otro medio tiene que ser autorizado por el director de la Revista Explorador Digital.
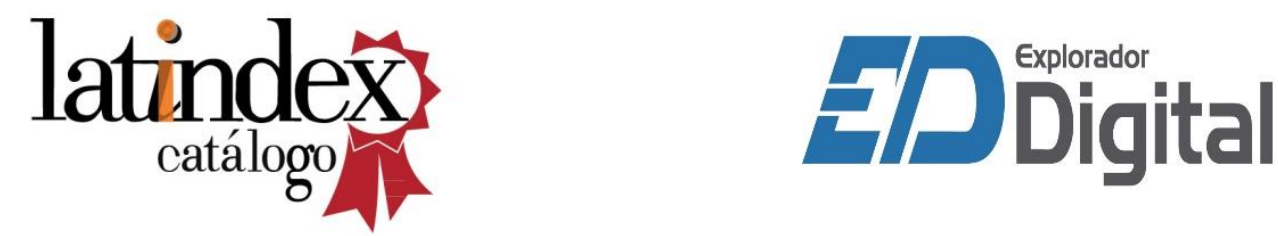